\title{
Active Tuberculosis in Children Receiving Chemotherapy
}

\author{
Ilham Tadmori*, Sarra Benmiloud, Mohamed Habibi, Mostapha Hida \\ Unité d'hémato-Oncologie, Service de Pediatrie, Hôpital Mère, Enfant, CHU Hassan II-Fès, Morocco \\ Email: hida63@yahoo.fr, ‘tadmori.ilham@hotmail.fr, ‘tadmori.ilham@gmail.com, \\ benmiloudsarra@yahoo.fr,mohamed.hbibi@hotmail.fr
}

How to cite this paper: Tadmori, I., Benmiloud, S., Habibi, M. and Hida, M. (2022) Active Tuberculosis in Children Receiving Chemotherapy. Open Journal of Pediatrics, $12,75-80$.

https://doi.org/10.4236/ojped.2022.121009

Received: November 2, 2021

Accepted: February 15, 2022

Published: February 18, 2022

Copyright (c) 2022 by author(s) and Scientific Research Publishing Inc. This work is licensed under the Creative Commons Attribution International License (CC BY 4.0)

http://creativecommons.org/licenses/by/4.0/

\begin{abstract}
Tuberculosis infections is a serious illness and can be life threatening for patients with hematologic malignancies. We are reporting the observations of two children aged 7 and 5 years undergoing anticancer treatment for hematological malignancies. The symptoms were, in the first case, cough, purulent sputum and signs of tuberculosis impregnation, for the second case, cervical adenopathies (PDA) with inflammatory signs. The evolution under antituberculosis treatment was favorable for both patients, with a 5-year follow-up for the first patient and one year for the second case. The tuberculosis did not affect chemotherapy. The goal of our work is to specify the etio-pathogenic mechanisms, the clinical, therapeutic and evolutionary particularities of this rare association.
\end{abstract}

\section{Keywords}

Hematologic Disease, Tuberculosis, Child, Chemotherapy

\section{Introduction}

Tuberculosis (TB) is a serious, life-threatening disease for patients with cancer. The cases of tuberculosis in pediatric oncology departments are rare. The occurrence of active tuberculosis in children with cancer treated with chemotherapy is rarely reported in the literature, there are only a few reports of Mycobacterium tuberculosis disease in children with oncologic or hematologic diseases [1] [2]. Patients with immunosuppression, due to underlying disease or drug-induced disease, are particularly susceptible to developing TB disease; patients with acute leukemia (AL) are predisposed to developing infections, including tuberculosis (TB) [3] [4] [5] [6].

Through these two observations collected over a period of ten years from 2011 to 2020, we are reporting the experience of the unit of pediatric oncology in the 
department of pediatrics at the CHU Hassan II Fez. The purpose is to specify, the etio-pathogenic mechanisms, the clinical particularities, evolution and therapeutic of this rare association.

Case $\mathrm{N}^{\circ} 1$ : Male child of 7 years old, vaccinated according to the national vaccination program with the scar of BCG. The family is from a low socioeconomic level and living in a rural area. The child was treated for Hodgkin's disease of the cervix and axillae with B signs (fever and general condition) classified as stage IIB unfavorable group and put under chemotherapy according to the MDH 2005 protocol. The chemotherapy is based on the administration of Vincristine, Doxorubicin, and Cyclophosphamide and corticosteroid therapy. The diagnosis of Hodgkin's disease was based on the anatomopathological study of the cervical lymph node biopsy which showed, follicular zones with clear germinal centres. The interfollicular zone is enlarged, and is covered by a polymorphic cell population consisting of epithelial cells, eosinophilic, lymphocytes and enlarged tumour cells with strongly nucleated vesicular nuclei. Some cells are binucleated giving a mirror-like appearance reminiscent of reed Sternberg cells. An IHC study was carried out and the tum cells expressed CD30 and CD15, CD20 and $\mathrm{CD} 3$ mark B and T cells in a reactionary manner. In conclusion: this is a mixed cellularity Hodgkin's lymphoma. In the history, we noted that the older sister was under an anti-bacillary treatment three months after the beginning of chemotherapy for our patient. Six months after the beginning of the treatment, that is to say after the sixth course of chemotherapy, the child presented a chronic cough evolving over three weeks associated with an alteration of the general state with weight loss of $3 \mathrm{~kg}$ and the reappearance of fever $\left(38.5^{\circ} \mathrm{C}\right)$ especially in the evening. The clinical examination showed no peripheral adenopathy, no hepatosplenomegaly. The child was put on broad-spectrum antibiotics with a biological and radiological check-up, given the suspicion of a relapse or another pathology associated with his condition (tuberculosis type, as his sister was under treatment). The chest X-ray showed a cavernous appearance (Figure 1).

The CT scan showed the appearance of an intra parenchymal cavitary lesion of the right inferior lobe and right para hilar, with a discreetly thickened irregular wall reaching $04 \mathrm{~mm}$ in places, with hydroaerobic content presenting two thickened endo-luminal partitions seeming to communicate with the bronchi and measuring $64 \times 47 \mathrm{~mm}$ in diameter. It was associated with a peri-lesional condensation with a ground-glass appearance and millimetric micronodules near a centro-lobular disposition and the appearance of some upper and lower mediastinal, right latero-tracheal, sub carinal and latero-esophageal adenopathies (ADP) measuring for the most voluminous one $13 \mathrm{~mm}$ in minor axis in the right latero-esophagus presenting a necrotic center (Figure 2, Figure 3).

The blood count showed lymphopenia at 400 elements $/ \mathrm{ml}$ with leukocytes at 5400 elements $/ \mathrm{ml}$ and neutrophils at 3530 elements $/ \mathrm{ml}$ and the sedimentation rate (SV) at $80 \mathrm{~mm}$ the first hour. The intradermal tuberculin test was positive with an induration diameter of less than $12 \mathrm{~mm}$. The bacilloscopy study of sputum by gastric tubing came back in favour of the presence of BAAR (acid-fast bacilli). 


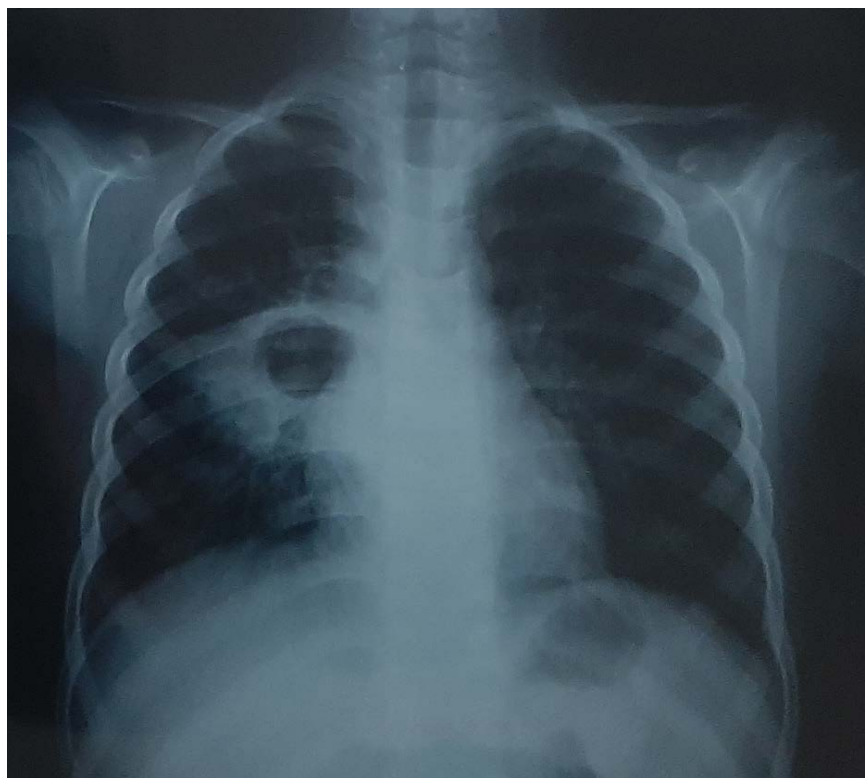

Figure 1. A chest X-ray of 7 year-old, showing an aspect of a cavern during diagnosis of TB.

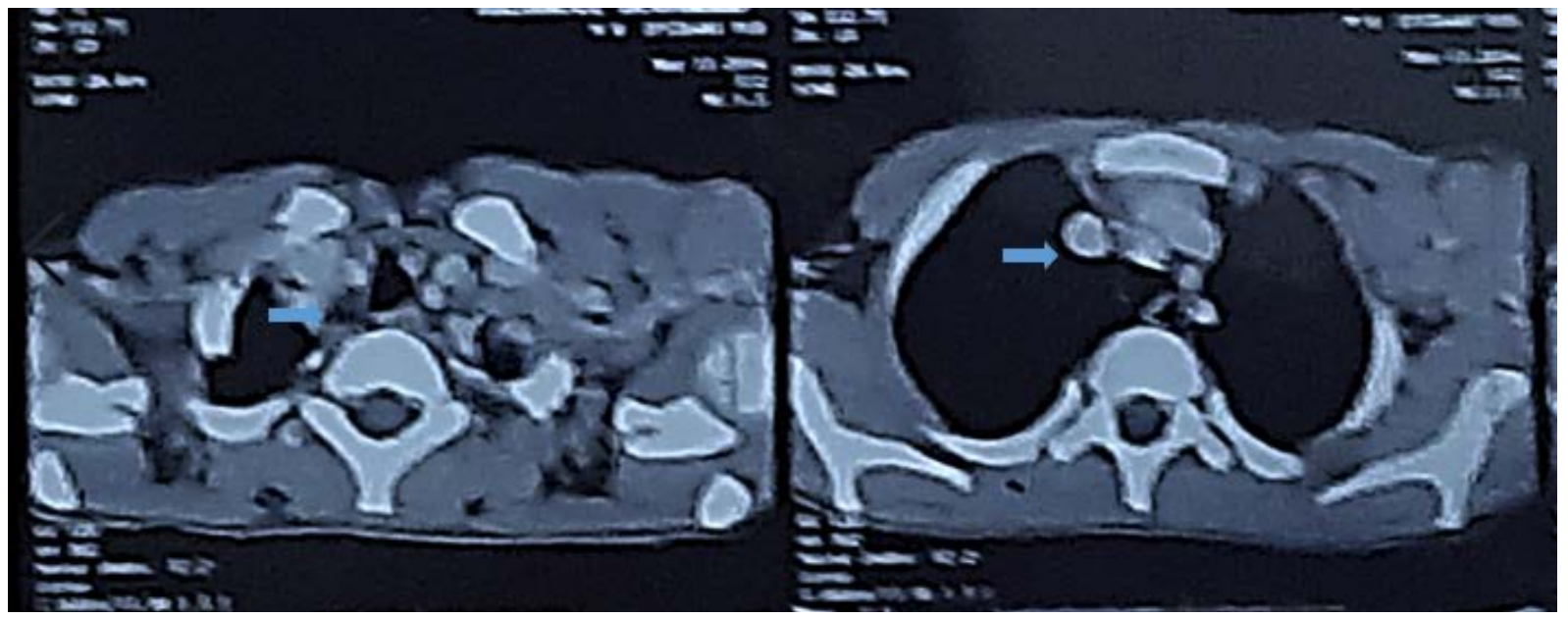

Figure 2. CT images of 7 year-old, showing right upper mediastinal, subcarinal and latero-esophageal adenopathies with a necrotic centre.
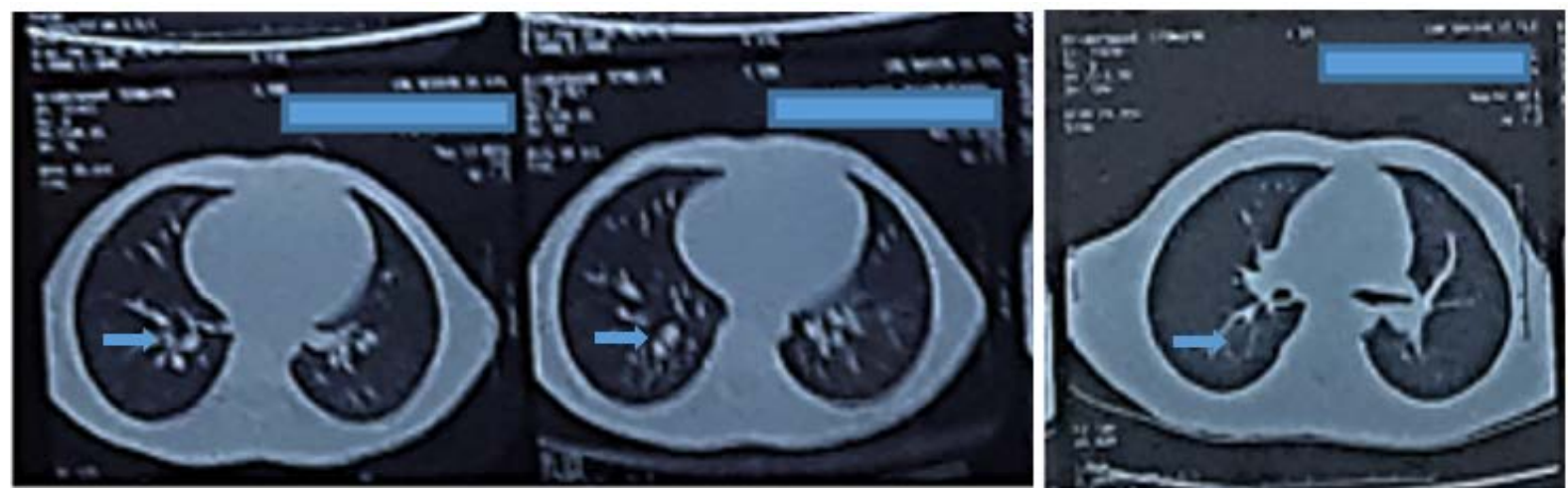

Figure 3. CT images of 7 year-old, showing Intraparenchymal cavitary lesion of the right inferior lobe and right para hilar, with hydroaerobic content. Peri-lesional condensation with frosted glass and millimetric micronodules in the vicinity with a centro-lobular disposition. 
The attitude was to stop chemotherapy for two weeks and to start anti bacillary treatment with the 2RHZ/4RH (Rifampicins + Isoniazid + Pyrazinamide/Rifampicins + Isoniazid) regimen. The patient was in remission and was successfully followed with a 6-month anti-tuberculosis treatment with episodes of hepatic cytolysis not exceeding 5 times the normal without any complication with an event-free follow-up of four years after the end of treatment.

Case $\mathrm{N}^{\circ} 2$ : Child aged 5 years, with no notable pathological history, notably no tuberculosis contagion and vaccinated according to the national vaccination program with the BCG scar in place. The family is of average socio-economic level and lives in the city. The child is followed for acute lymphoblastic leukemia classified as Standard Risk treated according to the MARALL 2006 protocol, under maintenance chemotherapy based on 6 Mercaptopurine and low doses of weekly Methotrexate. He presented 20 months after the beginning of his treatment a multiple right cervical adenopathy (ADP) progressively increasing in volume, evolving over five weeks associated with fever peaks quantified at $38^{\circ} \mathrm{C}$ $39^{\circ} \mathrm{C}$. The clinical examination showed a $6^{*} 4 \mathrm{~cm}$ hard and fixed ADP with inflammatory signs opposite without any other clinical signs. Initially, a relapse was suspected, but the workup came back negative. An infectious work-up showed an accelerated SV of 120 in the first hour and the blood count showed lymphopenia of 792 elements $/ \mathrm{mm}^{3}$. The patient was put on broad-spectrum antibiotic therapy but without improvement. The cervical ultrasound showed a right laterocervical ADP, a true mass, with poly-lobed contours, hypoechoic, containing liquid areas with necrosis, measuring $2.8 \times 4.8 \times 5 \mathrm{~cm}$. The intradermal tuberculin test was positive at $10 \mathrm{~mm}$. The sputum study by gastric tubing came back negative. Chest X-ray was unremarkable. An adenectomy was performed and the anatomopathological study came back in favor of a lymph node tuberculosis with presence of caseous necrosis. Antibacillary treatment was started with the $2 \mathrm{RHZ} / 4 \mathrm{RH}$ regimen with a transitory stop of chemotherapy for three weeks. The patient was in remission and was successfully treated for 6 months with episodes of liver cytolysis not exceeding 3 times normal without any complication with a one-year follow-up after the end of treatment.

\section{Discussion}

Cancer and tuberculosis, the main cause of morbidity and mortality, is a major public health problem in the world. However, tuberculosis in children with cancer is not a commonly described infection [7]. All circumstances of immune deficiency, due to underlying disease or drug-induced, favor the development of tuberculosis in patients with immunosuppression, and they are susceptible to developing TB disease [1] [4] [5] [8] [9] [10]. Children with solid cancers or hematologic malignancies have a high relative risk of developing $\mathrm{TB}$ and should be considered for routine screening and treatment of latent $\mathrm{TB}$, especially when they come from settings with a high incidence of TB [1] [9]. Patients with malignancies, particularly those with hematologic malignancies (HM), are at higher 
risk of developing TB than the general population [4] [5] [6] [7] [9]. Immunosuppression is a condition characterizing the long and evolving course of all subjects with and followed for cancer, which may be secondary to the neoplastic pathology and/or anti-cancer chemotherapy. The natural defense against tuberculosis requires predominantly a cell-mediated immune response. The higher incidence of tuberculosis in patients with hematologic malignancies is attributed primarily to impaired cellular immunity [4] [9]. This impaired immunity is secondary to the use of corticosteroids as well as chemotherapy products, which would explain the susceptibility of these patients to develop TB disease [1] [4] [8] [9]. It is estimated that the relative risk of tuberculosis in patients with hematologic diseases is 2 to 40 times higher than in the general population [4] [8] [11] [12]. Clinical symptoms are much less specific in malnourished, immunocompromised children or those with HIV or malaria. Diagnosis of tuberculosis is more difficult in young children and immunocompromised patients, as both populations are less likely to develop a positive tuberculin skin test, and less likely to have positive sputum staining for fibro-bronchial aspirates. Childhood tuberculosis usually presents with fever, cough, adenopathy, loss of appetite, and weight. Atypical presentations are also reported in children and adults [5] [13]. Mycobacterium tuberculosis is an important pathogen in patients with malignant diseases and should be promptly investigated for the disease if clinically suspected. The management of mycobacterial infection in children with cancer is a challenge, since the treatment of tuberculosis in the context of ongoing immunosuppression is difficult and published guidelines are not available. In adults, the response to TB treatment does not differ significantly between immunocompromised and immunocompetent adults. A good clinical response to TB therapy has been reported in adult patients with underlying hematologic malignancies treated with isoniazid, rifampicin, pyrazinamide, and ethambutol for 2 months, followed by isoniazid and rifampicin for 4 to 10 months [13].

However, according to our presentation of the two cases of tuberculosis in our two patients undergoing chemotherapy for their hematological malignancy, neither the clinical presentation nor the diagnosis nor the therapeutic protocol of tuberculosis disease was an obstacle to anti-cancer treatment, especially in patients living in endemic areas such as Morocco.

\section{Conclusion}

Because tuberculosis is a serious infection in immunocompromised persons, empirical antituberculosis treatment is sometimes necessary when clinical and radiological features strongly suggest tuberculosis, especially in patients living in endemic areas. In children, cancer chemotherapy is not a barrier to treatment of TB disease.

\section{Conflicts of Interest}

The authors declare no conflicts of interest regarding the publication of this paper. 


\section{References}

[1] van de Lool, K., et al. (2018) Lymphnode Tuberculosis in a 4-Year-Old Boy with Relapsed Ganglioneuroblastoma: A Case Report. BMC Infectious Diseases, 18, Article No. 105. https://doi.org/10.1186/s12879-018-3016-x

[2] Al-Anazi, K.A., Al-Jasser, A.M. and Evans, D.AP. (2007) Infections Caused by Mycobacterium Tuberculosis in Patients with Hematological Disorders and in Recipients of Hematopoietic Stem Cell Transplant, a Twelve Year Retrospective Study. Annals of Clinical Microbiology and Antimicrobials, 6, Article No. 16. https://doi.org/10.1186/1476-0711-6-16

[3] Gauchon, A., et al. (2008) Stratégie de Prise en Charge de Contages Tuberculeux Dans un Service d'oncologie-pédiatrique. Archives de Pédiatrie, 15, 236-244. https://doi.org/10.1016/j.arcped.2008.01.005

[4] Anibarro, L. and Pena, A. (2014) Tuberculosis in Patients with Haematological Malignancies. Mediterranean Journal of Hematology and Infectious Diseases, 6, e2014026. https://doi.org/10.4084/mjhid.2014.026

[5] Jain, A., et al. (2018) Analysis of Clinical Profile and Outcome of Tuberculosis in Patients with Acute Leukemia. Indian Journal of Hematology and Blood Transfusion, 34, 430-442. https://doi.org/10.1007/s12288-017-0875-Z

[6] Panda, S., et al. (2015) Disseminated Tuberculosis Presenting as Prolonged Fever without Source in a Pediatric Patient with Acute Lymphoblastic Leukemia. Journal of Cancer Research and Therapeutics, 11, 1043.

https://doi.org/10.4103/0973-1482.154030

[7] Mishra, P., et al. (2006) Tuberculosis in Acute Leukemia: A Clinico-Hematological Profile. Hematology, 11, 335-340. https://doi.org/10.1080/10245330600915818

[8] Billy, C. And Perronne, C. (2004) Aspects Cliniques et Thérapeutiques de la Tuberculose Chez l'enfant et l'adulte. EMC-Maladies Infectieuses, 1, 81-98. https://doi.org/10.1016/j.emcmi.2004.01.002

[9] Baka, M., et al. (2013) Successful Treatment in a Child with Anaplastic Large Cell Lymphoma and Coexistence of Pulmonary Tuberculosis. Hindawi Publishing Corporation Case Reports in Pediatrics, 2013, Article ID: 928701. https://doi.org/10.1155/2013/928701

[10] Dobler, C.C., et al. (2017) Risk of Tuberculosis in Patients with Solid Cancers and Haematological Malignancies: A Systematic Review and Meta-Analysis. European Respiratory Journal, 50, Article ID: 1700157. https://doi.org/10.1183/13993003.00157-2017

[11] Stefan, D.C., et al. (2008) Tuberculosis in Oncology Patients. Annals of Tropical Paediatrics, 28, 111-116. https://doi.org/10.1179/146532808X302125

[12] Cheng, M.P., et al. (2017) Risk of Active Tuberculosis in Patients with Cancer: A Systematic Review and Meta-Analysis. Clinical Infectious Diseases, 64, 635-644.

[13] Zivanovic, S., et al. (2010) A Case of Acute Tuberculous Pleuropneumonia in a Patient with Acute Lymphoblastic Leukemia. The Scientific World Journal, 10, 578 585. https://doi.org/10.1100/tsw.2010.52 